\title{
VERITAS long-term (2006-2014) observations of the BL Lac object 1ES 0806+524
}

\section{Matteo Cerruti* for the VERITAS Collaboration}

Harvard-Smithsonian Center for Astrophysics, 60 Garden St., Cambridge, MA 02138, USA

E-mail: matteo.cerruti@cfa.harvard.edu

\begin{abstract}
The discovery of the high-frequency-peaked BL Lac object 1ES 0806+524 ( $\mathrm{z}=0.138)$ as a source of very-high-energy (VHE, E>100 GeV) gamma-ray photons was announced in 2008 by the VERITAS Collaboration, at a level of $1.8 \%$ of the Crab Nebula flux above $300 \mathrm{GeV}$. Since then, VERITAS has continued observing the source over multiple seasons, significantly improving the significance of the detection. We report the results of the analysis of the 2006-2014 VERITAS data, corresponding to a total exposure of about 70 hours. We present the new, average VHE spectrum of the source, together with the multi-year light-curve constraining long-term VHE variability.
\end{abstract}

The 34th International Cosmic Ray Conference,

30 July- 6 August, 2015

The Hague, The Netherlands

\footnotetext{
* Speaker.
} 


\section{Introduction}

Blazars are the most common source of $\gamma$-ray photons in the extra-galactic sky. The current generation of imaging atmospheric Cherenkov telescopes (IACTs), observing the universe at veryhigh-energies (E>100 GeV; VHE), has identified around 60 blazars as VHE emitters ${ }^{1}$. Among the different blazar subclasses (BL Lac objects and flat-spectrum radio-quasars, FSRQ; further divided into low/intermediate/high-synchrotron peaked blazars), the VHE extra-galactic sky is largely dominated by high-synchrotron-peaked BL Lac objects. A peculiarity of VHE astronomy is that the universe is opaque to VHE photons, due to pair-production triggered by the interaction of $\gamma$-rays with the extra-galactic background light (EBL) in visible and infrared light. The effect is that VHE astronomy is limited in redshift, the current most distant VHE source being the gravitationallylensed blazar $S 30218+35$, at $\mathrm{z}=0.94$. This implies that the majority of VHE blazars are nearby high-synchrotron-peaked BL Lac objects.

The variability properties of VHE blazars are still poorly understood, some blazars being stable sources of VHE photons, while others having been detected only during rapid flaring episodes. The VERITAS collaboration is carrying on a long-term monitoring of several VHE blazars [1], with the goal of studying the long-term evolution of the VHE flux, possibly associated with spectral variability.

The BL Lac object $1 E S 0806+524$ was one of the first discoveries of the current generation of IACTs, detected by VERITAS during observations in 2006-2008 [2]. In this work, we present the results of VERITAS observations of $1 E S 0806+524$ from 2006 to 2014, re-analyzing as well part of the data published in [2].

During February 2011, the MAGIC collaboration has reported the detection of a rapid (day-long) flare from 1ES 0806+524, as discussed in [3]. The VERITAS telescopes observed the source before and after the MAGIC flare, and we present as well the detailed results of the VERITAS observations during 2011.

\section{VERITAS data analysis}

VERITAS (Very Energetic Radiation Imaging Telescope Array System) is an array of four 12meter diameter telescopes, located at the base of Mount Hopkins, in Southern Arizona, at the Fred Lawrence Whipple Observatory. The array is arranged in a diamond configuration, with sides of about 100m. Each telescope is equipped with a camera made of 499 photo-multiplier tubes, sensitive to the weak Cherenkov light emitted by the cascades produced in the interaction of VHE photons with the Earth's atmosphere.

The system is able to reconstruct $\gamma$-ray emission from about $85 \mathrm{GeV}$ to $>30 \mathrm{TeV}$, with an energy resolution of $15-25 \%$. The field of view of the instrument is $3.5^{\circ}$, with an angular resolution $R_{68 \%}<0.1^{\circ}$ at $1 \mathrm{TeV}$. The system is able to detect a source with a flux level comparable to the Crab nebula in 12 minutes, and a source with a flux level of the order of $5 \%$ of the Crab nebula in 1.5 hours.

\footnotetext{
${ }^{1}$ See tevcat.uchicago.edu for an updated list of VHE sources
} 
The VERITAS array, in operation since 2006, has gone through several upgrades during its scientific operations: in 2009 one of the four telescopes was relocated in order to get a symmetric array; in 2012 the PMTs in the camera have been replaced, achieving a better sensitivity and a lower energy threshold. For further details see [4, 5].

The observations of $1 E S 0806+524$ presented in this proceedings include data taken before/after the relocation of one of the telescopes, and before/after the camera upgrade. No observations taken under bright-moonlight conditions [6,7] are included in the data-set. The data-set comprises 72 hours of VERITAS exposure (after taking into account the dead-time of the instrument). Compared to the results presented in [2], we excluded from the data-set the observations taken in 2006 using only two of the four VERITAS telescopes.

All observations were taken using the wobble observing mode, where the telescopes are pointed $0.5^{\circ}$ away from the target, to allow a simultaneous estimation of the emission from the source and the background. The discrimination between Cherenkov showers triggered by $\gamma$-rays or cosmic rays is done using standard cuts on the images, as described in [4].

For the data-set as just described, the VHE emission from 1ES 0806+524 is detected with the VERITAS instrument at a level of 7.1 standard deviations over the background, as estimated following [8], above an energy threshold of $300 \mathrm{GeV}$.

\subsection{Spectral reconstruction}

The previously published VHE spectrum of $1 E S 0806+524$, as presented in [2], was parametrized by a power-law function between 300 and $700 \mathrm{GeV}$ :

$$
\Phi(E)\left[\mathrm{cm}^{-2} \mathrm{~s}^{-1} \mathrm{TeV}^{-1}\right]=K\left(\frac{E}{400 \mathrm{GeV}}\right)^{-\Gamma}
$$

with $K=(6.8 \pm 1.7) \times 10^{-12} \mathrm{~cm}^{-2} \mathrm{~s}^{-1} \mathrm{TeV}^{-1}$ and $\Gamma=3.6 \pm 1.0$.

Using the 2006-2014 data-set, the new average VERITAS spectrum of IES 0806+524 extends from $300 \mathrm{GeV}$ to $1.8 \mathrm{TeV}$, and can be parametrized by a power-law function

$$
\Phi(E)\left[\mathrm{cm}^{-2} \mathrm{~s}^{-1} \mathrm{TeV}^{-1}\right]=K\left(\frac{E}{600 \mathrm{GeV}}\right)^{-\Gamma}
$$

with $K=(1.4 \pm 0.3) \times 10^{-12} \mathrm{~cm}^{-2} \mathrm{~s}^{-1} \mathrm{TeV}^{-1}$ and $\Gamma=3.4 \pm 0.6$. The new result is consistent with the previous VERITAS result, but extends to higher energies, and has smaller uncertainties. The errors on the spectral reconstruction are statistical only. In Figure 1 we show the average spectrum of $1 E S 0806+524$ as measured by VERITAS.

\subsection{Temporal variability}

In Figure 2 we show the VHE integral light-curve of $1 E S$ 0806+524, estimated above the VERITAS energy threshold $(300 \mathrm{GeV})$. In the top panel we present the long-term (2006-2014) 


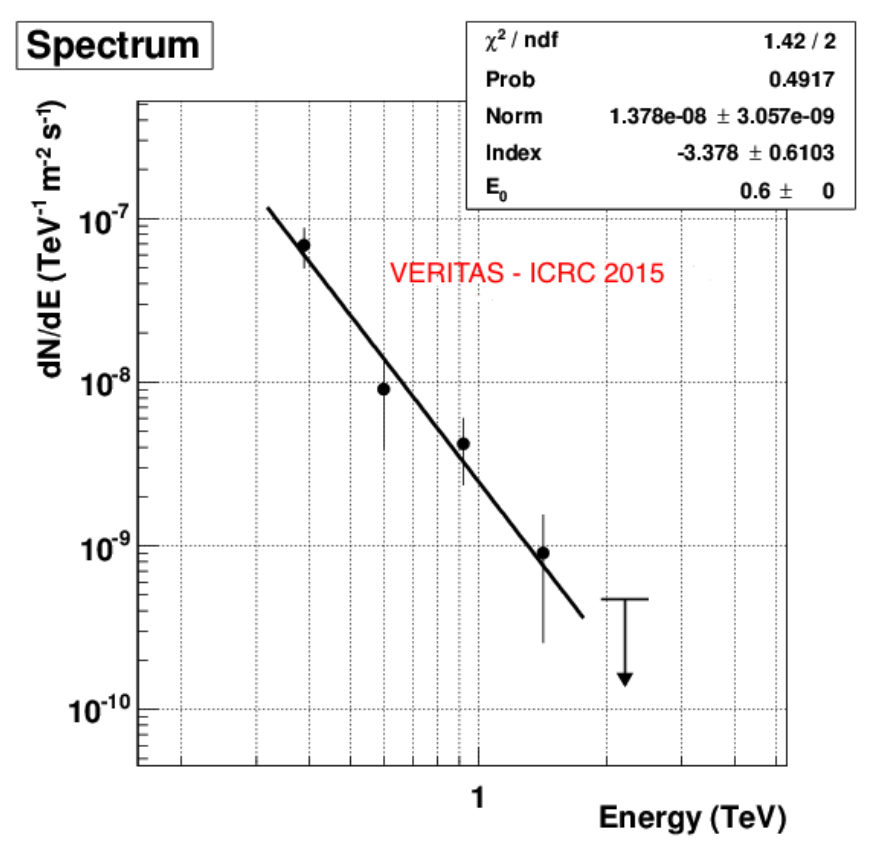

Figure 1: Spectrum of 1ES 0806+524 as measured by VERITAS.
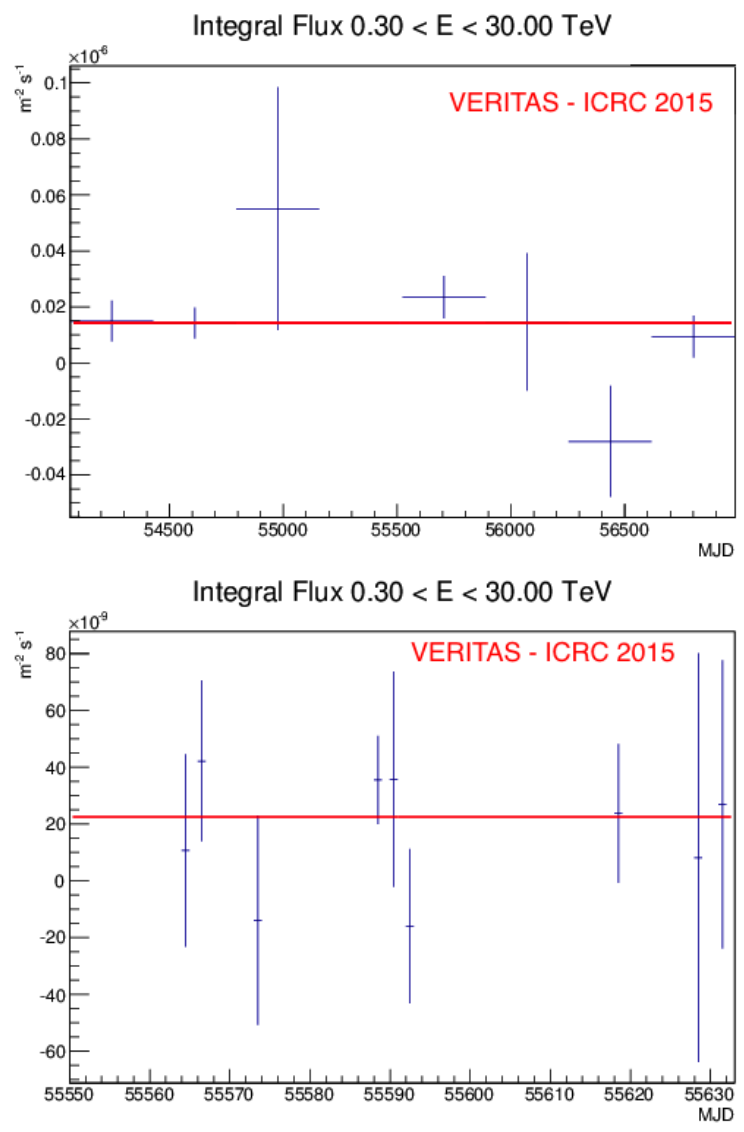

Figure 2: Light-curve of IES 0806+524 as measured by VERITAS. Top: year-by-year light-curve, from 2006 to 2014; Bottom: day-by-day light-curve, zoomed on the 2011 season. 
light-curve rebinned per observing season (September to June). No evidence of variability is seen in the data: the flux from $1 E S 0806+524$ is compatible with constant emission $\left(\chi^{2}=7 / 6\right)$, and the average integral flux is $(1.4 \pm 0.3) \times 10^{-12} \mathrm{~cm}^{-2} \mathrm{~s}^{-1}$, corresponding to $1.2 \% \pm 0.3 \%$ of the flux from the Crab nebula [9].

During February 2011, IES 0806+524 showed rapid VHE flaring activity, as reported in [3]. The VERITAS telescopes observed the source before and after the MAGIC flare, showing a flux in agreement with the average VHE emission. The day-by-day VERITAS light-curve of 1ES 0806+524 during 2011 is shown in the bottom panel of Fig. 2. This result supports the fact that the $\gamma$-ray flare observed by MAGIC was an isolated event, happening on a short time-scale.

\section{Conclusions}

In this work we reported the results of long-term (2006-2014) observations of the BL Lac object 1ES 0806+524 using the VERITAS telescopes. The flux and spectrum of the source is consistent with the previous VERITAS publication, which used data from 2006 to 2008, but the higher statistics allowed a better spectral reconstruction, extending to higher energies. The long-term light-curve is consistent with a constant flux, at a level of about $1.2 \%$ of the Crab nebula flux above $300 \mathrm{GeV}$. We also investigated in detail the daily light-curve during the 2011 observing season, before and after the VHE flare observed by the MAGIC telescopes, without detecting any evidence of enhanced emission. This result supports the fact that the February 2011 flare seen by MAGIC was characterized by a short time-scale, on the order of a day.

\section{References}

[1] Dumm J., Proceedings of the 33rd ICRC, arXiv 1308.0287

[2] Acciari et al., ApJ, 690, L126, 2009

[3] Aleksic et al., MNRAS, 451, 5258, 2015

[4] Holder et al., Astroparticle Physics, 25, 391, 2006

[5] Kieda, D., Proceedings of the 33rd ICRC, arXiv 1308.4849

[6] Griffin, S. these proceedings

[7] Cerruti, M. these proceedings

[8] Li and Ma, ApJ, 272, 317, 1983

[9] Hillas et al., ApJ, 503, 744, 1998 